\title{
Identification and Analysis of Radio Monitoring Service Based on the Meet-in-the-Middle Strategy
}

\author{
Fan Jing ${ }^{1}$, Li Huiyu ${ }^{1+}$, Fei Wei ${ }^{2}$, Luo Chao ${ }^{3}$ \\ The State Radio Monitoring Center, Beijing 100037, China.
}

\begin{abstract}
Based on a deep understanding of SOA and services, different service identification methods are compared, radio monitoring atomic services and business application services are identified and analyzed by using the meet-in-the-middle strategy in this paper. This identification and analysis method can provide reference for other departments or industries in the construction of application systems based on SOA architecture.
\end{abstract}

Keywords: SOA, service identification, meet-in-the-middle strategy, radio monitoring

\section{Introduction}

In recent years, the third-generation information technology featuring mobile Internet, cloud computing and big data has flourished. In the new era, using advanced information technology to achieve the integration, automation and intelligence of radio management is an effective way to improve the radio monitor and manage capacity.

For a long time, due to the diversity and the wide geographical distribution of radio monitoring equipment, the interconnection and data sharing among different monitoring systems have been difficult.In order to solve the problems to achieve flexible interconnection, efficient collaboration and rapid sharing of various systems, as well as data collection, integration, analysis and utilization, a radio management integration construction idea was proposed, and an application integration support platform that satisfies the integration between applications was preliminarily established based on the service-oriented architecture (SOA)[1].

How to identify flexible, efficient, and reusable monitoring services from the abstract business applications is a basic, important and difficult task in the integrated construction of radio monitoring[2][3][4]. Based on the research of service identification strategies and radio monitoring application requirements, and in combination with monitoring equipment and system functions, the radio monitoring services are first identified effectively based on the meet-in-the-middle strategy.

\section{Service Identification Strategy}

\subsection{Considerations}

Service identification is a crucial step in the implementation of the SOA cycle. Good service identification can make better use of the advantages of SOA. In the SOA, service is the most core abstraction means, which has a clear function and usually encapsulates business functions or data. Service granularity is the size of the functions that a service contains. Fine-grained services provide relatively small functional units, or exchange a small amount of data. The advantage is it has strong flexibility, but the completion of complex business logic often requires the organization of a large number of fine-grained services, it is less

\footnotetext{
+ Corresponding author. Tel.: 18610498625; fax: 010-68009299.

E-mail address: seu_lhy@163.com.
} 
efficient. Coarse-grained services encapsulate large blocks of business / technical capabilities in an abstract interface. The advantage is the number of service request interactions is reduced and the cost is reduced, but the flexibility and reusability of service calls is also reduced. Therefore, services are not as fine-grained as possible, nor as coarse-grained as possible, but to find a balance among them to obtain the benefits of reduced costs and flexibility[5][6].

Three aspects need to be considered when identifying and designing a service with the right granularity:

- Reusability: Reusability is the ability of a service to be applied in different contexts. Fine-grained services are easier to reuse, but if a set of functions will not be called apart, there is no need to split this set of functions into multiple services.

- Flexibility: Flexibility is the ability to easily make changes based on circumstances. One goal of SOA is to make IT more agile and to adapt more quickly to a continuously changing business environment. Fine-grained services can be assembled more easily, providing more flexibility for delivering new business functions or changing business processes, but the good organization, efficiency, development and maintenance costs of backstage service need to be considered.

- Performance: The complexity of the service, and the number of service calls to implement a business activity are the main aspects that affect SOA performance. When the service granularity is coarser, the business logic will be more complex and the response to the client will be slower. When the service granularity is finer, the efficiency of the single service execution will be higher, but the number of service calls to implement a business activity will be more, and the performance overhead will increase significantly.

In summary, service identification and analysis should be performed according to the actual needs of business activities. Service functions should meet business orientation and business rules, and the services should evolve iteratively, even be refactored to adapt the business change.

\subsection{Strategies}

In the current research on service identification, the commonly used service identification strategies are top-down and bottom-up [5][7].

- Top-down approach, is also known as domain decomposition approach. This approach starts with business analysis and decomposes business processes into unit business activities layer by layer. The top-level business domain model is the input of the decomposition. According to the detailed description of the business domain model, the business domain can be subdivided according to the business responsibilities. Each node on the business activity tree obtained through stepwise decomposition will be the candidate for service.

- Bottom-up approach, is also known as existing asset analysis approach. The purpose of this approach is to use existing assets to implement services. Existing assets include: existing systems, packages or custom applications, industry specifications or business models. The service candidates can be verified or discovered by analyzing the existing assets, and the feasibility of service implement decisions can also be verified as early as possible by analyzing the technical limitations of existing systems, packages, or custom applications, which provides an important basis for service realization decisions.

The above two identification approaches have their advantages and disadvantages. The top-down approach focuses on identifying services at the business level. In this way, a high-quality service architecture can be formed usually, but it is very time-consuming and pays less attention to the technical-level factors of the service. The bottom-up approach focuses on identifying services at the technical level, and pays less attention to the reusability of services and subsequent business requirements. In many cases, the services are simply added to the existing application environment, there is no service-oriented architecture analysis in fact.

The meet-in-the-middle strategy can achieve better practical results than simply applying one approach. On the one hand, the key target activities can be discovered from the analysis of business processes and abstracted to services through top-down approach. On the other hand, the key program functions can be discovered from the analysis of existing business systems, as shown in Fig. 1. In this paper, according to the 
requirements of radio monitoring business and the functions of monitoring system, radio monitoring services are identified and analysed by using the meet-in-the-middle strategy.

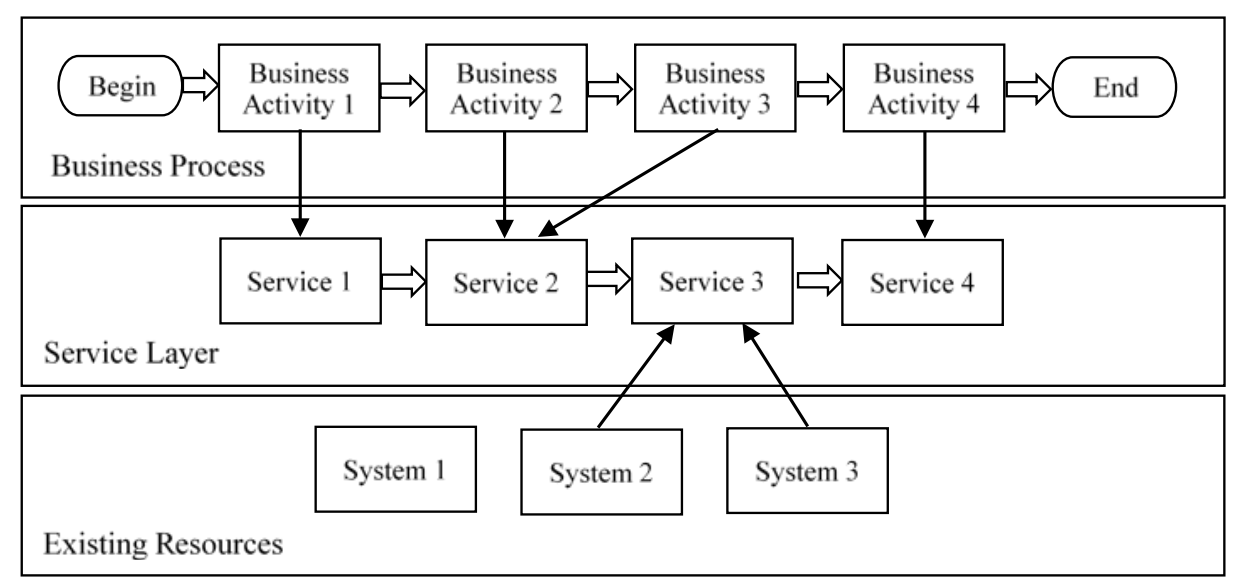

Fig. 1: Meet-in-the-Middle Service Identification Strategy.

\section{Identification and Analysis of Radio Monitoring Service}

There are many kinds of radio monitoring equipment and application systems, they have different manufacturers and technical standards, different operation modes and data formats, wide geographical distribution, so the interconnection and data sharing between different monitoring systems are difficult. How to realize the interconnection between different equipment and systems, realize the decoupling of monitoring equipment and upper monitoring application, adapt to the expansion of new equipment and the change of new business development flexibly, make full use of existing resources and reduce the system repeated construction are the main problems need to be solved in radio monitoring integration. As the first step of integration construction, service identification plays a vital role in design of service-oriented architectural.

Considering the purpose of radio monitoring service identification and analysis, the radio monitoring service identification and analysis is divided into four steps based on the meet-in-the-middle service identification strategy:

- Business demand analysis: The monitoring business work mode need to be analyzed deeply to further identify the business application requirements and services.

- Business process analysis: Identify the business process based on the deep understanding of the application requirements.

- Identify services from the business process: Identify the atomic services that make up the business process based on the deep understanding of the business process.

- Identify services from the system functions: Identify the functions that exposed to support services from existing monitoring systems, and map the services to potential service providers.

The first three steps mainly adopt the top-down identification approach, and the last step mainly adopts the bottom-up identification approach.

\subsection{Business Demand Analysis}

In this paper, the radio monitoring tasks and business requirements are analysed comprehensively base on years of monitoring experience. And the content of each business requirement is analysed to identify the relevant business application services.

For example, the "UHF signal monitoring task" in radio monitoring applications aims to comprehensively grasp the characteristics and transmission laws of existing radio signals, detect illegal signals and new stations in time, strengthen the verification of unknown signals to further enrich the monitoring database provides data support for the effective management of frequency and stations. The "automatic signal census", "signal modulation analysis", "designated frequency point positioning", "designated frequency band positioning" and other business application services are identified by requirements analysis, as shown in Fig. 2. 
Business application services identified from the different monitoring tasks may be duplicated. By summarizing all identified business application services and removing duplicate services, a total business application service list can be obtained.

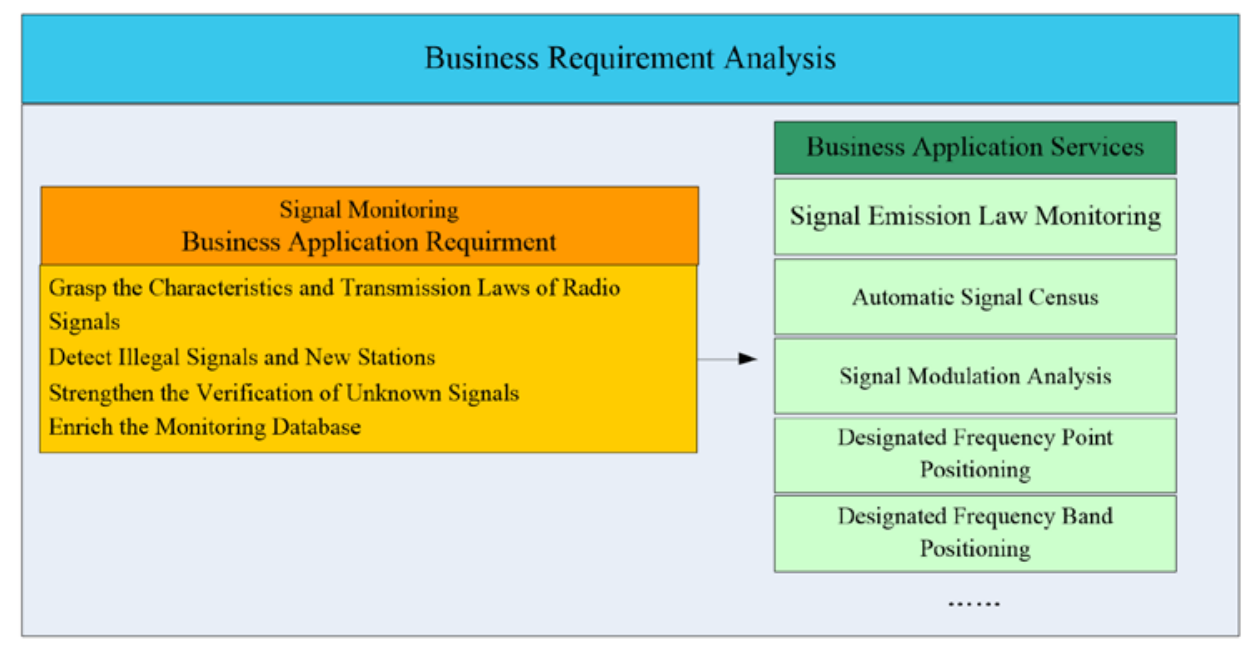

Fig. 2: Business Demand Analysis Schematic of the "UHF Signal Monitoring".

\subsection{Business Process Analysis}

Based on the deep understanding of the radio monitoring business requirements, the business process is defined for each identified business application service.

For the "automatic signal census" business application service identified above, further analysis of the business process is carried out. Firstly, a device with the function of frequency sweep signal interception is chosen, and need to work at the specified frequency band to intercept the signals, then extraction results are compared to the monitoring database, if the signal is unknown, further monitoring and direction-finding equipment is needed to measure the parameters of the unknown signal, and the verification results are stored in the database and reported. The business flow chart is shown in Fig. 3.

\subsection{Identify Services from the Business Process}

Based on the deep understanding of the business process, the atomic services needed to make up the business process are identified.

For the "automatic signal census" business, according to its business process, the "monitoring equipment and antenna connection assignment", "frequency sweep signal interception", "level measurement", "bandwidth measurement", "field intensity measurement", "single frequency direction finding" or "intermediate frequency FFT direction", "signal audio recording", "IQ data acquisition", "spectrum data collection", "monitoring database comparison ", "station database comparison", "ITU database comparison" and other atomic services are identified, as shown in Fig. 3.

Services identified in multiple business processes may be duplicated, which is also consistent with the reusability characteristic of SOA services. Summarizing all the services identified by the above process, the "top-down" service identification is completed.

\subsection{Identify Services from the System Functions}

To ensure the feasibility of the identified services, the functions of all existing and under construction monitoring systems and equipment are combed, and atomic services provided by these systems are refined, then corresponds to the services identified by "top-down" approach, the services are mapped to a potential service providers.

Continue the above example, by mapping analysis, the "monitoring equipment and antenna connection assignment" service provided by the antenna switching matrix, the "frequency sweep signal interception", "level measurement", "bandwidth measurement", "field intensity measurement", "signal audio recording", "IQ data acquisition" and "spectrum data collection" services provided by the fixed monitoring system, the "single frequency direction finding" and "intermediate frequency FFT direction" services provided by the 
fixed direction finding system, the "monitoring database comparison ", "station database comparison", "ITU database comparison" services respectively provided by the monitoring database, station database and ITU database, as shown in Fig. 3.

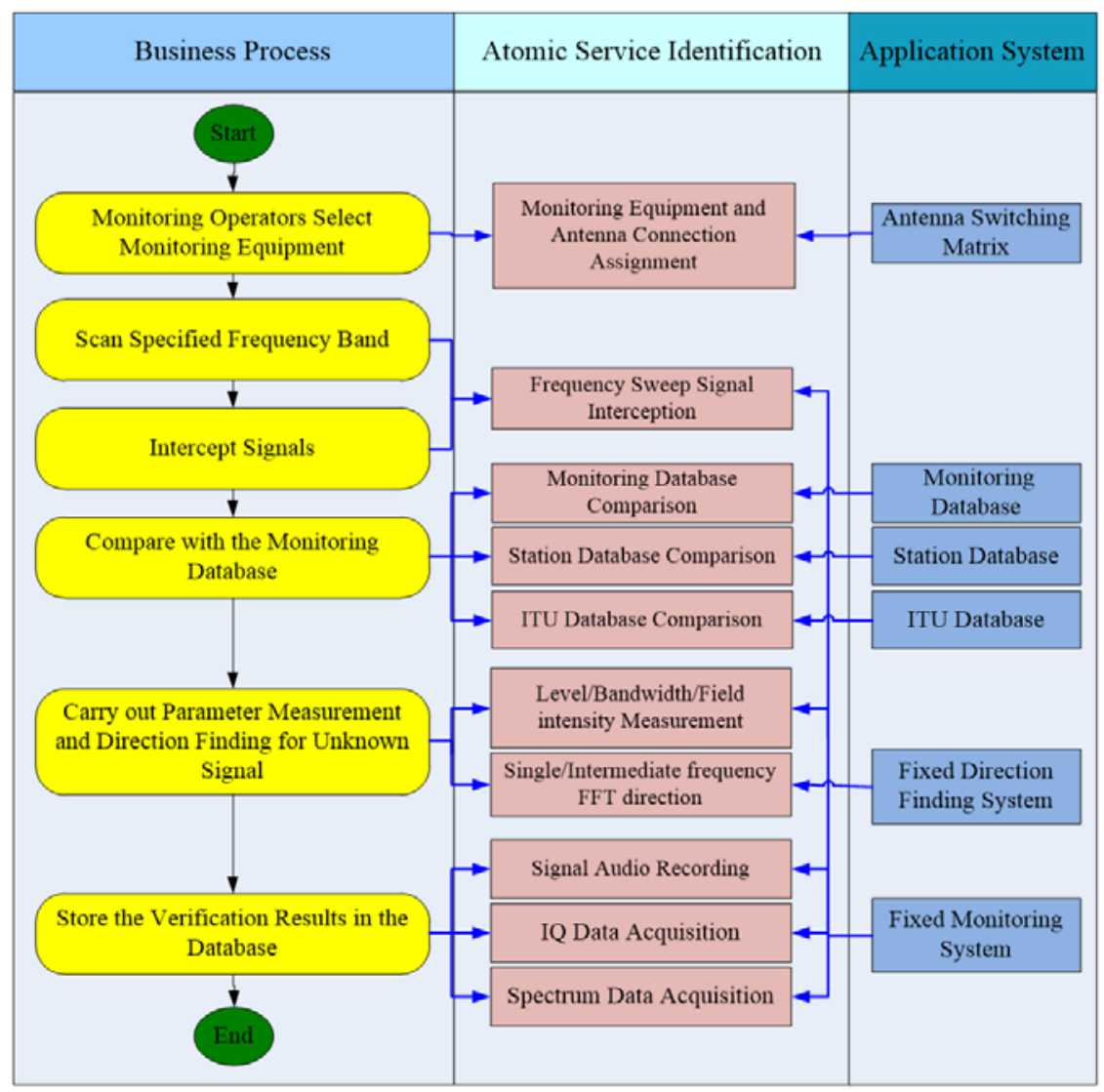

Fig. 3: Schematic Diagram of "Automatic Signal Census" Business Process and Service Identification.

By further orchestrating atomic services and supplementing some analysis logic and algorithms, business application services (composite services) are formed, which is the basis of realizing the monitoring automation and intelligence. Fig. 4 shows the flow chart of the "signal automatic census" business application service obtained while identifying the monitoring atomic services.

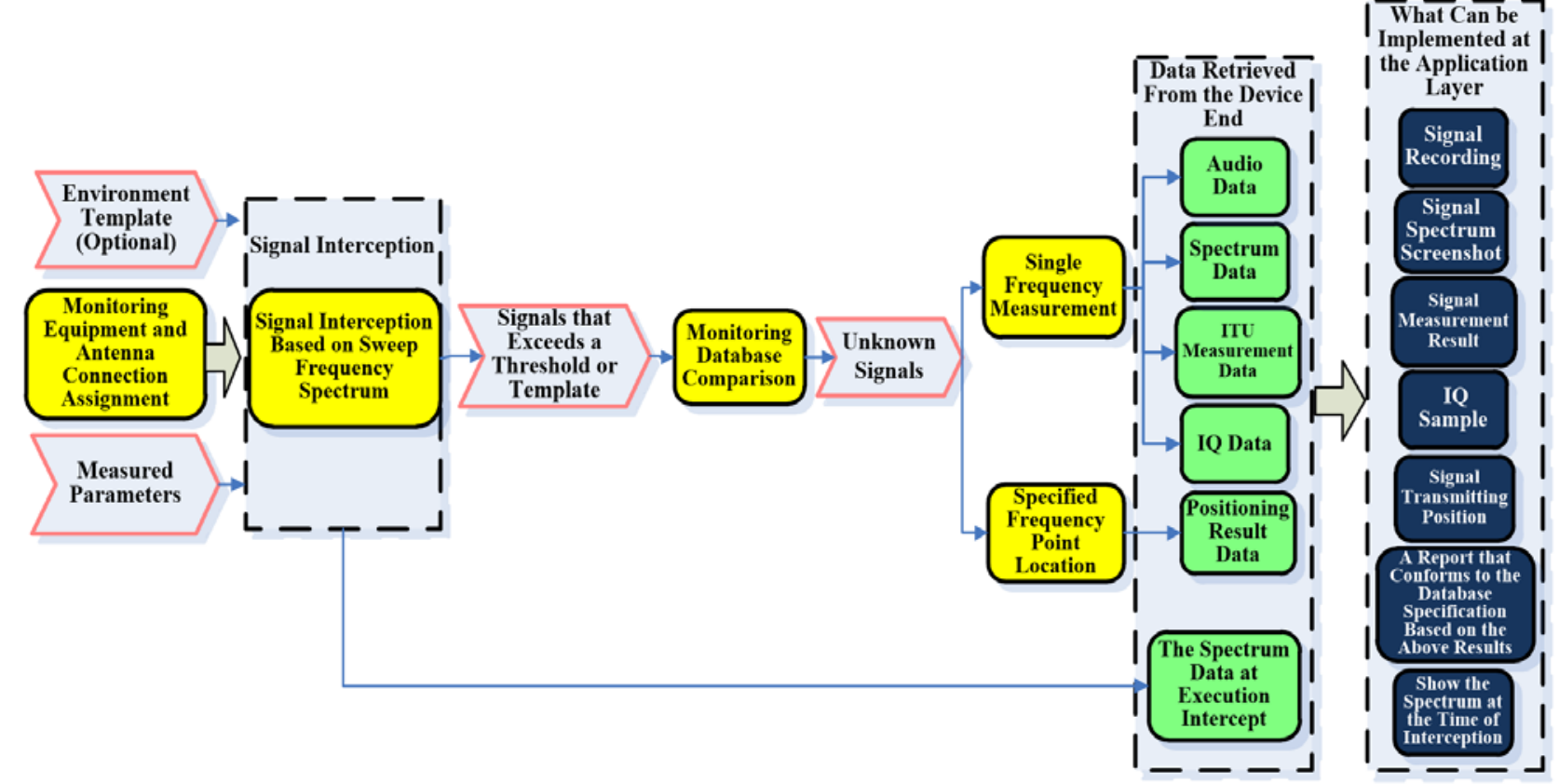

Fig. 4: Schematic Diagram of "Automatic Signal Census" Service (Composite Service) flow. 


\section{Result and Discussion}

In the work of radio monitoring service identification and analysis, we have the following experiences.

- In many cases, people often adopt the bottom-up identification approach, simply implement service delivery after a business system is developed. This approach is easy to implement, also can form a certain set of services, but the service reusability is poor, the advantages of SOA architecture are not really realized. Therefore, it is recommended to conduct a comprehensive service-oriented analysis first in the construction of application systems based on SOA architecture.

- Comprehensive service-oriented analysis of the business requires that service identification person be very familiar with the business. As the organizer and manager of radio monitoring tasks, we have many years of front-line monitoring work experience and are very familiar with monitoring services and monitoring equipment. Even so, in this work, we repeatedly discussed with equipment manufacturers and integrators, conducted pilot verification tests to ensure the realization of services and application efficiency.

- When using the meet-in-the-middle strategy for service identification, it is necessary to find a balance point during the alignment, but it's not easy and requires repeated adjustments and optimizations. The operation of identifying services from business requirements and refining services from system functions is not completely separated, but is a process of mutual integration and constant adjustment.

- The meet-in-the-middle strategy can effectively ensure the reusability and flexibility of the identified services, and the optimization of service performance can be achieved by continuously verifying and adjusting the service.

\section{Summary}

Service identification and analysis is a key step in system construction based on SOA architecture. In the integrated construction of radio monitoring, using the meet-in-the-middle strategy introduced in this paper, 27 business application services and 107 atomic services commonly used in HF, satellite and UHF monitoring are identified, and the relevant service interface specifications are formulated. Radio monitoring services are not limited to these, along with the different needs of the business as well as the application system updates, more business application services and atomic services can be obtained by using the analysis method in this paper, which can also provide reference to the services identification in integrated construction for other units or departments.

\section{References}

[1] Fan Jing, Li Huiyu, Fei Wei, ,et al. The Construction of an Integrated Platform to Achieve a New Leap in Radio Monitoring. China Radio. 2016.

[2] Anurag Shashwat, Deepak Kumar. A Service Identification Model for Service Oriented Architecture. 3rd International Conference on Computational Intelligence \& Communication Technology (CICT). 2017.

[3] Jens Weller, Werner Esswein, Jeannette Stark, et al. Meet the Challenge in Service Identification: A Ratio-based Approach. Pacific Asia Conference on Information Systems (PACIS) 2009 Proceedings, 2009.

[4] Neelavathi, Dadapeer, K. Vivekanandan. Service Selection Based on Status Identification in SOA. 3rd International Conference on Electronics Computer Technology. 2011, p.113-116

[5] Johan denn Haan. SOA and Service Identification. Information on: http://www.theenterprisearchitect.eu/archive/2007/04/26/soa-and-service-identification.

[6] Padmal Vitharana, Hemant Jain, Fatemeh Zahedi. Strategy-Based Design of Reusable Business Components. IEEE Transactions on Systems, Man, and Cybernetics-Part C: Applications and Reviews. 2004, 34(4): p.460-474.

[7] Thomas Erl. Service-Oriented Architecture Concepts, Technology, and Design. Prentice Hall. 2005. 